\title{
Predicting the Infectious Disease Spread Using Floating Population Data in Seoul, South Korea
}

\author{
Jinhwa Jang ${ }^{1,2}$ and Insung Ahn ${ }^{1,3 *}$ \\ ${ }^{1}$ Biomedical Prediction Technology Laboratory, Korea Institute of Science and \\ Technology Information, 245 Daehak-ro, Yuseong-gu, Daejeon 34141, Korea \\ ${ }^{2}$ Laboratory of Computational Biology and Bioinformatics, Graduate School of \\ Public Health, Seoul National University, 1 Gwanak-ro, Gwanak-gu, Seoul 08826, \\ Korea \\ ${ }^{3}$ Dept. of Big Data Science, University of Science \& Technology, 217 Gajeong-ro, \\ Yuseong-gu, Daejeon 34113, KOREA \\ 1,2 jhjang@kisti.re.kr, ${ }^{1,3 *}{ }^{*}$ isahn@kisti.re.kr
}

\begin{abstract}
Emerging of global infectious diseases threat to worldwide induced numerous patients through person to person infection. In previous study, we investigated effects of traveling nationwide using expressway data on the spread of H1N1 influenza virus in Korea during 2009-2010. As a result, influenza epidemic patterns of the 2009 were correlated with some region traffic flow. In this study, we focused on Seoul which region is a highest density of population in Korea. Also using system dynamics-based simulation of the spread of an infectious disease in each of the 25 districts in Seoul was performed. Consequently, the decrease in the number of infected people in the district with a large floating population size was more significant than that with a high population density. This study is meaningful as it visualized the number of infected people on the map, which includes actual geographical information and the changes over time in the number of individuals that belong to $S, I$, and $R$ classes of population. Also mathematical model based on Korea unique traffic and population movement information could be used. These results can be applying additional population and traffic data in the future, and support making decisions when establishing an effective infectious disease control strategy.
\end{abstract}

Keywords: System Dynamics Modeling, Infectious disease, Floating Population Data, Census Data, Simulation

\section{Introduction}

Infectious diseases spread rapidly or chronically over wide areas, causing infection or death of several people and creating worldwide chaos on issues such as those associated with medical resource allocation [1]. About 50 million people worldwide were killed during 1918-1920 because of the Spanish flu pandemic caused by H1N1 influenza A virus, and approximately 36.9 million people died of acquired immune deficiency syndrome (AIDS) due to human immunodeficiency virus (HIV), which was first discovered in the United States in 1960 [2,3]. Ebola virus infection, a recent global issue, spread rapidly in Africa during 2014-2015. According to the WHO's Ebola Situation Report, 28,616 people were infected by the virus, and 11,310 died by March 2016 [4]. Additionally, Zika virus, which has become the most serious public health issue of 2016, is a mosquito-borne virus that has been reported to spread to 42 countries. Zika virus was first detected in Africa; however, it has now spread to other countries such as the Americas including Brazil. Zika virus is particularly a severe threat to pregnant women 
since it causes microcephaly in the fetus by attacking cortical neural precursors, which create the cerebral cortex or surface of the brain where neurons are concentrated while the fetal brain is developing [5].

Therefore, it is necessary to establish a foundation that can block the movement of disease vectors by assessing transmission routes based on research; hence, the spread of infectious diseases that cause numerous human, economic, and social problems could be predicted and checked. A mathematical model for the transmission of smallpox developed by Daniel Bernoulli in 1760 initiated the development of mathematical models for predicting the spread of diseases. Daniel Bernoulli conducted research to assess smallpox vaccination programs, and his model is applicable to all diseases, which people become immune to after surviving an infection [6]. In 1906, Hamer developed a discrete model for measles. This model was the first to assume that the number of disease outbreaks per unit time is related to the mathematical product of the density of healthy people (susceptibles), who could be infected by a pathogen, and infected people (infectives). In 1926, Kermack and McKendrick introduced a concept of disease threshold, which suggests that the density of people susceptible to a disease needs to surpass a certain threshold value for a disease to spread for the first time [7]. Therefore, predicative modeling of infectious diseases was focused on predicting the transmission of diseases among humans. Individuals are classified into M, S, E, I, and R classes according to the stage of disease development: $M$ (passive immune) class represents a stage where IgG antibodies produced from the mother, who has an infectious disease, are transferred to the fetus, enabling it to develop passive immunity; $\mathrm{S}$ (susceptible) class corresponds to a stage in which one is likely to get infected since antibodies are absent in the system; E (exposed) class refers to a stage in which an individual, who was infected in S class, is in the latent period in which his ability to infect other individuals has not yet expressed; I (infected) class denotes a stage in which an individual is infected and can infect other individuals; and lastly, $\mathrm{R}$ (recovered) class represents the last stage of infection in which an individual is in good health by acquiring permanent immunity to the disease. Furthermore, various mathematical predictive models, such as SI, SIS, SIR, SEIR, SEIRS, SIRS, SEI, SEIS, MSEIR, and MSEIRS, can be created through various combinations of the classes [8].

There are several simulation modeling methods for predicting the spread of infectious diseases, such as discrete event modeling, agent-based modeling, and system dynamics modeling. Discrete event modeling is a method that classifies events according to the time order and performs simulations based on each event [9]. Agent-based modeling is a method to perform simulations by defining agents and determining each of their behaviors; subsequently, it involves connecting agents and exposing them to interaction or dynamic environment [10]. System dynamics modeling is a method to create a computer model based on dynamic and cyclical causal relations and to investigate how a phenomenon composed of complicated causal relations changes dynamically with time [11]. For instance, Pruyt analyzed the 2009 flu pandemic and analyzed changes in the number of infected people by using a system dynamics model based on the SIR model [12]. Additionally, several predictive studies of the spread of infectious diseases were conducted based on the feedback structure between level and flow that display the state of a system; based on a system dynamics model, it was shown how contagious diseases such as severe acute respiratory syndrome (SARS), Middle East respiratory syndrome (MERS), avian influenza, and HIV are transmitted among humans [13]. It is important to input accurate values for key variables, the level and flow variables, in such system dynamics-based predictive studies on the spread of infectious diseases. A study simulated the introduction of infectious diseases by using actual data; for example, a simulation was carried out in the United States using the three basic reproduction numbers of 55 international metropolitan cities with regard to the emergence and spread of an influenza virus in the U.S. during February, 2009 based on the actual aerial network. The initial 
time of the infectious disease introduction to the country was based on the patterns of aerial network and dynamic characteristics of infectious disease. The infection rate and location where the disease was first observed were predicted [14].

Accordingly, a correlation analysis was conducted in this pilot study to investigate effects of traveling nationwide using expressways on the spread of H1N1 influenza virus in Korea during 2009-2010. Further, the analysis was based on the time when the first patient infected with the virus was reported. Therefore, dissemination patterns of the 2009 influenza pandemic were correlated with some traffic conditions; it was proved through a data-based analysis that movements of a population through expressways could be utilized as indicators to predict the spread of an infectious disease [15]. We conducted a predictive study of the spread of an infectious disease based on the S-I-R system dynamics model in Seoul that has the highest population density in Korea; we utilized the floating population data of 25 districts (gu) in Seoul based on the "2014 Seoul Floating Population Survey".

\section{Materials and Methods}

\subsection{Epidemiological System Dynamics Modeling}

The S-I-R model, one of the basic models for studying the spread of infectious diseases can calculate changes in the values of $S$, I, and $R$ classes in $N$, that is, the whole population group, based on various parameter values. In the SIR model, infect individuals (I) get better at a certain rate, after transmitting the disease to healthy individuals (S), and recover $(\mathrm{R})$ from the disease and develop immunity against it. The individuals that belong to $\mathrm{R}$ become immune and can no longer get infected or transmit the disease. In this model, infectives (I) disappear with time and all individuals can either belong to $\mathrm{S}$ or $\mathrm{R}$ class. The equation of S-I-R model is as follows [7]. Among the variables used in the equation, B denotes a contract rate, $1 / \gamma$ denotes an average infectious period, and $N$ denotes the total population.

$$
\begin{gathered}
\frac{d S}{d t}=-\frac{\beta S I}{N} \\
\frac{d I}{d t}=-\frac{\beta S I}{N}-\gamma I \\
\frac{d R}{d t}=\gamma I
\end{gathered}
$$

In this study, a system dynamics model applied with the SIR equation was created for each of the 25 districts in Seoul as shown in Figure 1. Parameter values that influence the predicted result values of the spread of an infectious disease in the SIR model are the total population size, number of people who were first infected, infection rate, average infectious period, recovery rate, and number of contactable individuals per patient. In this study, the values reported by the WHO and Korea's census data with respect to outbreak of the H1N1 influenza pandemic in 2009 were applied to parameters. Additionally, the number of contactable individuals per patient, that is, 1.53 was obtained from the data on the outbreak of the 2009 influenza virus; this was adopted for reproduction value [14]. In cases of additional parameter values, 2 days were applied to latent incubation time, 0.95 to percentage of immunized agents, 0.9 to percentage of recovered agents, 0.14 to percentage of dead agents, 5 days to minimum time of dead agents, 14 days to maximum time to be recovered, and lastly, Korea census data were referred to for total population size. In this study, a system dynamics model was created for each of the 25 districts in Seoul, and by entering a census datum value of each district, the results of $S, I$, and $R$ 
classes and changes in the number infected patients were simulated on a map in which graphs were connected with the Geographical Information System (GIS).

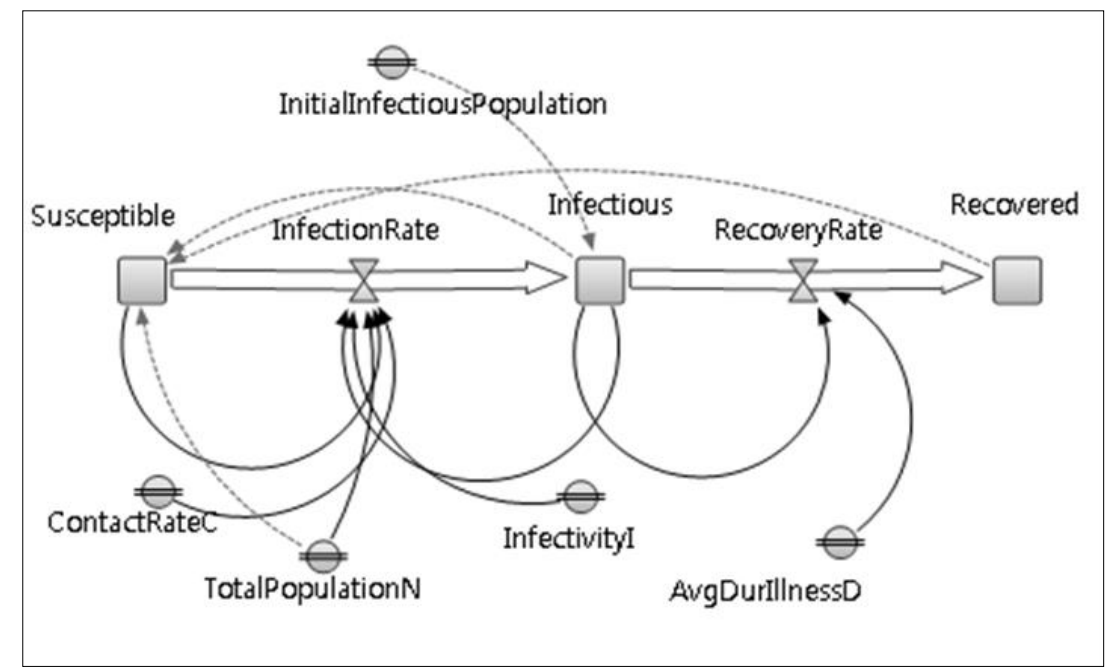

Figure 1. System Dynamics Flow of SIR Modeling

\subsection{Epidemic Scenario using Floating Population Data and Census Data}

Data on the population density of Seoul in 2014 provided from the Seoul Statistics Information System (http://stat.seoul.go.kr/) were referred to for obtaining information about population density and floating population size, and "Report of an Investigation into the Population of Seoul in 2014" provided by the Seoul Information Communication Square (http://opengov.seoul.go.kr) was referred to for obtaining information about floating population district wise (per/14hr). Seoul's district wise population was obtained by dividing the population size of a district by the total area. The size Seoul's floating population, according to "Report of an Investigation into the Population of Seoul in 2014", was obtained by counting pedestrians that passed from left to right across the reference line in front of a surveyor every 5 minutes between 07:30 and 19:30 hours in 1,000 representative areas and 111 major spots in Seoul. A result value was obtained by calculating the number of pedestrians for 14 hours. In this study, the data based on weekly average of floating population for a district, among the investigation results of floating population, were used. According to an investigation of the population of Seoul in 2014, Jung-gu (Jung District) had the highest weekly average floating population count among the 25 districts with 15,791 (per/14hr). It was followed by Jongno-gu, 10,386 (per/14hr); Dongjak-gu, 8,359 (per/14hr); Gwanak-gu, 6,797 (per/14hr); and Seodaemun-gu 6,660 (per/14hr). Seongbuk-gu had the lowest number of 2,943 (per/14hr). In this study, in order to apply the above data of floating population (district wise) to the previously designed SI-R model as per districts ( 25 districts in Seoul), a contact rate $(\beta)$ among the parameters used in the S-I-R model was considered. A contact rate refers to the number of close contacts per infected individual and was calculated by multiplying the number of close contacts per day per infected individual with the number of days of infection. Because a daily contact rate as per district is determined by a population density as per district, the result of the multiplication between population density and floating population size was applied. The values of population density and floating population size as per districts used in this study are as shown in Table 1. 


\section{Table 1. Application of the Contact Rate Using Census Data and floating} Population Data by 25 Districts in Seoul, Korea

\begin{tabular}{|c|c|c|c|c|c|}
\hline District & Den. Pop & Flo. Pop. & District & Den. Pop & Flo. Pop. \\
\hline GN & 14,728 & 6,656 & SDM & 18,351 & 6,660 \\
\hline GD & 18,842 & 6,327 & SC & 9,601 & 5,809 \\
\hline GB & 14,172 & 4,453 & SD & 18,093 & 3,815 \\
\hline GS & 14,376 & 3,924 & SB & 19,104 & 2,943 \\
\hline GA & 17,891 & 6,797 & SP & 19,704 & 4,015 \\
\hline GJ & 21,987 & 4,643 & YC & 28,098 & 3,526 \\
\hline GR & 22,596 & 5,650 & YDP & 17,033 & 4,553 \\
\hline GC & 19,675 & 4,547 & YS & 11,337 & 5,583 \\
\hline NW & 16,317 & 3,548 & EP & 16,920 & 4,126 \\
\hline DB & 17,056 & 4,209 & JoN & 6,851 & 10,386 \\
\hline DDM & 26,301 & 4,040 & Ju & 13,486 & 15,791 \\
\hline DJ & 25,240 & 8,359 & JuN & 22,633 & 2,848 \\
\hline MP & 16,706 & 5,117 & Total & 17,013 & 5,533 \\
\hline
\end{tabular}

Den. Pop, Density of population(per/ $\mathrm{km}^{2}$ ); Flo. Pop, Floating population(per/14hr); GN, Gangnam-gu; GD, Gangdong-gu; GB, Gangbuk-gu; GS, Gangseo-gu; GA, Gwanak-gu; GJ, Gwangjin-gu; GR, Guro-gu; GC, Geumcheon-gu; NW, Nowon-gu; DB, Dobong-gu; DDM, Dongdaemun-gu; DJ, Dongjak-gu; MP, Mapo-gu; SDM, Seodaemun-gu; SC, Seocho-gu; SD, Seongdong-gu; SB, Seongbuk-gu; SP, Songpa-gu; YC, Yangcheon-gu; YDP, Yeongdeungpo-gu; YS: Youngsan-gu; EP, Eunpyeong-gu; JoN, Jongno-gu; Ju, Jung-gu; JuN, Jungnang-gu

\subsection{Simulation of Infectious Disease Spread in Seoul using AnyLogic}

In this study, for the system dynamics-based simulation of the spread of an infectious disease, the Professional edition was used for GIS map integration in AnyLogic 7, a multimethod simulation software, that supports all discrete event models, agent-based models, and system dynamics models. First, a system dynamics S-I-R model of the entire Seoul was designed for main simulation, and changes in population according to time variable are shown in a graph. In the map, the entire territory of Seoul is marked, and a regional compartment by district is shown. The changes in time zone are expressed in red with respect to the changes in the number of infected individual by district. Additionally, by designing an S-I-R model for each of the 25 districts, contact rate values applied with total population and floating population sizes were inputted. When a simulation is performed based on this, it is possible to observe changes in the population size, which belongs to S, I, and R classes, in case of an outbreak of an infectious disease with respect to time. Additionally, by connecting with Seoul's GIS map, changes in the number of infected individuals district wise and according to time were expressed in red to differentiate from the numerical changes in infectious people with respect to time and district wise. Further, based on simulation results, the most effective plan for combating the outbreak of an infectious disease was selected by observing changes in the number of patients in Seoul, that is, in case a population migration rate of a specific district, where there is a rapid increase in the number of infected people, is decreased. 


\section{Results}

\subsection{Calculated Contact Rate using Floating Population Data in Seoul}

First, absence of correlation between the two variables, population density and floating population, was confirmed. The result of correlation analysis conducted by using the data of population densities and floating populations of the 25 districts of Seoul was $r=-0.375$ $(\mathrm{p}<0.05)$; this implies that each datum about population densities and floating populations needs to be applied separately to a mathematical model for predicting spread of the disease. Accordingly, in this study, a modified contact rate equation was developed to apply population density and floating population size by density together when calculating a contact rate $(\beta)$. The basic equation (1) for calculating a contact rate was modified to the equation (2) by applying floating population to (1). $\gamma$ used in calculating a contact rate $(\beta)$ refers to total number of contacts and $p$ refers to transmission risk. Because floating population (f) is estimated to have an effect on the total number of contacts, the floating population size by district (the 25 districts in Seoul) was multiplied with the basic contact rate equation.

$$
\begin{gathered}
\beta=\gamma \times \mathrm{p} \\
\beta^{r}=\gamma \times \mathrm{p} \times \mathrm{f}
\end{gathered}
$$

Figure 3 shows modified contact rate values that were calculated together with population per $\mathrm{km}^{2}$ and population that moved during 14 hours across the 25 districts in Seoul. Therefore, unlike other districts, the floating population values were higher than that of population density in Jongno-gu and Jung-gu. In contrast, the floating population value of Yangcheon-gu with the highest population density was lower than that of the other districts. With respect to the contact rate, in which both the foregoing values were applied, the average of all districts in Seoul was 9.4 per/day, and that of Dongjak-gu with high population density and Jung-gu with high floating population were 21.1 per/day and $21.3 \mathrm{per} /$ day, respectively. Nowon-gu had the lowest contact value with 5.6 per/day, which is about four times lower than that for Jongno-gu, which has the highest value. In particular, Yangcheon-gu had the highest population density of $28,098 \mathrm{per} / \mathrm{km}^{2}$, and the fastest spread of disease was observed in this district before the floating population size with respect to districts was applied; however, the actual size of floating population in Yangcheon-gu was 3,526 per/14hr, which was more than four times lower than that of Jung-gu $(15,791 \mathrm{per} / 14 \mathrm{hr})$, which had the largest floating population. Additionally, when a contact rate in Yangcheon-gu was calculated by applying floating population size alongside population density in this study, it was similar to the mean contact rate of the 25 districts (9.4 per/day) with 9.9 per/day. Therefore, a contact rate value increases with an increase in the population density and floating population size. 


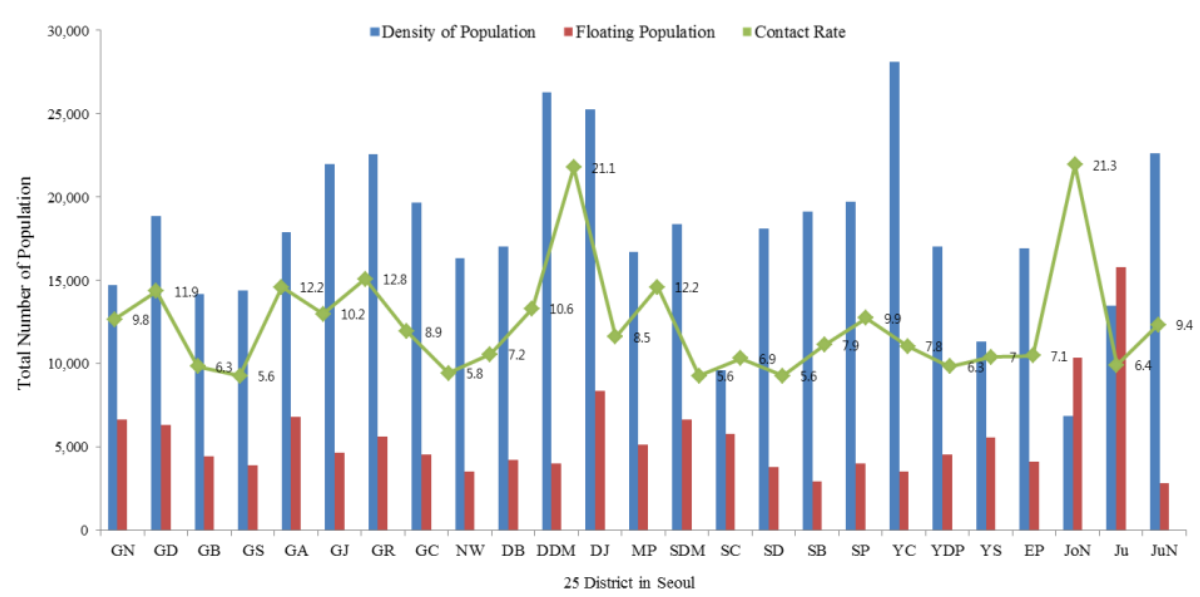

Figure 3. Calculated Contact Rate Using Floating Population data by 25 Districts in Seoul

GN, Gangnam-gu; GD, Gangdong-gu; GB, Gangbuk-gu; GS, Gangseo-gu; GA, Gwanakgu; GJ, Gwangjin-gu; GR, Guro-gu; GC, Geumcheon-gu; NW, Nowon-gu; DB, Dobonggu; DDM, Dongdaemun-gu; DJ, Dongjak-gu; MP, Mapo-gu; SDM, Seodaemun-gu; SC, Seocho-gu; SD, Seongdong-gu; SB, Seongbuk-gu; SP, Songpa-gu; YC, Yangcheon-gu; YDP, Yeongdeungpo-gu; YS: Youngsan-gu; EP, Eunpyeong-gu; JoN, Jongno-gu; Ju, Jung-gu; JuN, Jungnang-gu

\subsection{Infectious Disease Spreading Simulation in Seoul}

As a result of applying the SIR model by using the data on population densities and floating populations of the 25 districts in Seoul, the spread of an infectious disease increased faster than that observed in the other districts as the contact rate value increased with an increase in population density and floating population size. First, the fastest increase in the number of infected individuals was seen in Dongjak-gu and Jung-gu. The number of infected individuals increased and dissipated quickly in the order of Guro-gu, Gwanak-gu, and Seodaemun-gu. As a result of performing an S-I-R system dynamics simulation, based on the existing contact rate, on the total population in Seoul $(10,297,138)$ without applying floating population size, the maximum number of infected individuals was approximately 410,000 (3.98\% of the total population of Seoul) and was recorded during the sixth and seventh weeks after the first infected individual was discovered. The number of infected people gradually decreased over time, and infected individuals were not observed after the sixteenth week. Meanwhile, different trends were identified when a simulation was performed with respect to a contact rate, which was recalculated using the floating population data: the maximum number of infected individuals during the fifth and the sixth weeks after discovering the first patient was estimated at approximately 490,000 , which accounts for $4.75 \%$ of the total population in Seoul. With regard to the difference in the two simulation results, the number of infected people among the total population in Seoul was expected to increase by $0.77 \%$ when the floating population data were applied. 

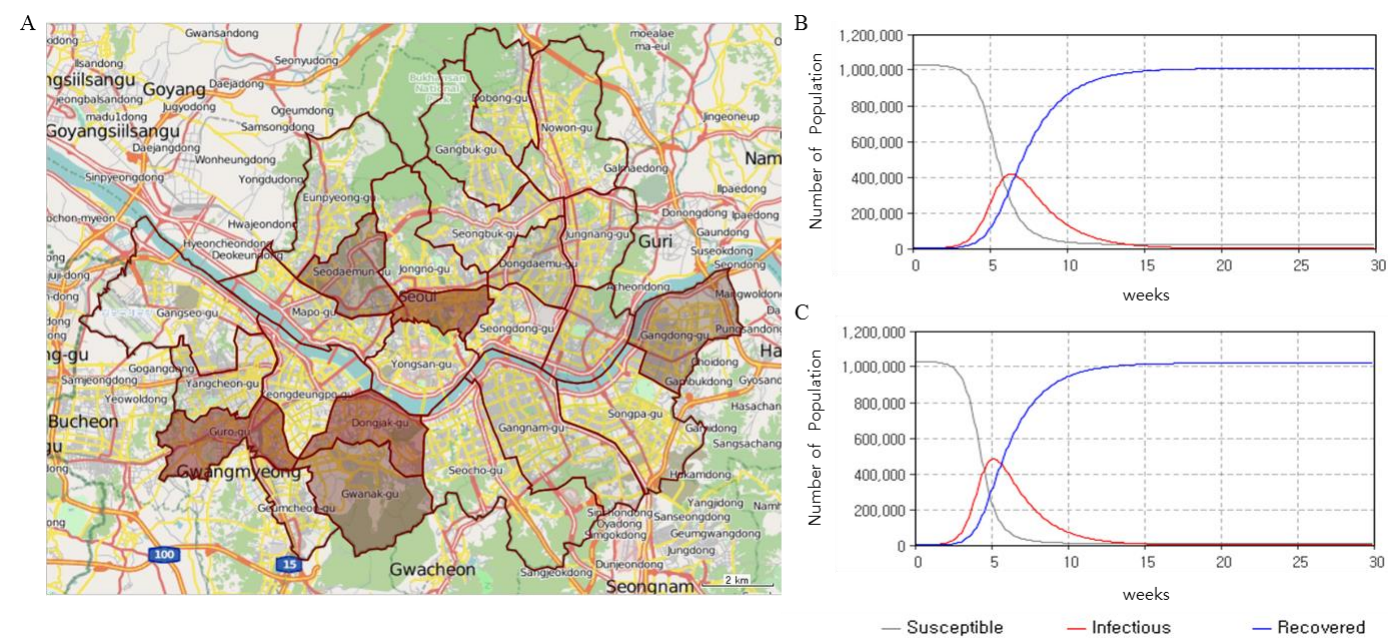

\section{Figure 4. Infectious Disease Spreading Simulation by 25 Districts in Seoul}

(A: disease spread simulation result on the map, B: SIR graph using $\beta$ in Seoul, C: SIR graph using $\beta$ ' in Seoul)

Here, a simulation was performed to verify the predicted number of infected individuals in Seoul while assuming that the contact rate will decrease after excluding $30 \%$ of floating populations in Jongno-gu (which had the largest floating population) and Jungnang-gu (which had the lowest floating population). Therefore, the maximum number of infected people among the total Seoul population was predicted as approximately 390,000 , that is, $3.7 \%$ of the entire Seoul population, when $30 \%$ of Jongno-gu's floating population was excluded. However, the number was predicted to be approximately 400,000 , that is, $3.88 \%$ of the entire Seoul population, when $30 \%$ of Jungang-gu's floating population was excluded. Therefore, it was proved that blocking people from moving in and out of a district with a high floating population is effective in decreasing the number of infected people in case of an outbreak of an infectious disease. Additionally, because Guro-gu had a high population density while Jongno-gu had a large size of floating population, a simulation was performed to determine which out of the two districts controlled the movement of floating population to obtain an even smaller number of infected individuals. Consequently, the maximum number of infected individuals was approximately 405,000 , that is, $3.93 \%$ of the entire Seoul population, when the movement of floating population was controlled with respect to Guro-gu, which is $0.05 \%$ higher than that observed with respect to Jongno-gu. It was proved that controlling the movement of floating population in a district with a large floating population size is more effective in decreasing the number of infected people than controlling parameters in a district with a high population density.

\section{Discussion and Conclusions}

We conducted a pilot study to predict the spread of an infectious disease by using Korea's unique information. First, the data about population and its movement via expressways were applied based on the statistics of traffic volume between expressway interchanges in Korea, and a comparative analysis was conducted with the prevalence of the 2009 H1N1 influenza pandemic. Consequently, the inflow and outflow of traffic between expressway interchanges along provinces in November 2009 and November 2014 were very different. Based on the correlation analysis with the incidence rate in 2009, a positive correlation with the volume of traffic in November 2009 was observed; 
though, it was not significant. Additionally, a negative correlation with the volume of traffic in 2011 was observed [15]. Therefore, an SEIR model was applied based on the data of census and traffic flow of 14 cities and provinces in Korea by using agent-based modeling-based AnyLogic 7. In particular, as a result of applying the SEIR model to Gangwon-do (Gangwon province), which had the largest volume of traffic in November 2009 , both numbers of I and E class individuals increased with an increase in the volume of traffic [15]. Therefore, it was confirmed that the movement of a population through expressways could be used as an actual predictive index for the spread of an infectious disease.

Subsequently, areas used in the simulation were narrowed down to the districts in Seoul with the highest population density in Korea, and a system dynamics-based simulation of the spread of an infectious disease in each of the 25 districts in Seoul was performed. In particular, simulation based on the modified contact rate was performed; the modification included multiplication of the floating population of each district with the mathematical product of the total number of contacts and transmission risks in order to apply the floating population data to the contact rate. It was confirmed that the spreading speed of an infectious disease was faster than that observed in other districts, and the contact rate value increased with a rise in the population density and floating population size. Additionally, in the two districts with high population density and large floating population size, respectively, the size of floating populations was reduced to investigate which out of the two districts would have a smaller number of infected people in case the in and out of the district movements were preemptively blocked. Consequently, the decrease in the number of infected people in the district with a large floating population size was more significant than that with a high population density. When an S-I-R system dynamics simulation was performed based on the entire Seoul population $(10,297,138)$ by applying floating population, the maximum number of infected people between the fifth and sixth weeks after discovering the first patient was predicted to be approximately 490,000, which makes up $4.75 \%$ of the total Seoul population. It was revealed that the number of infected people was higher than when floating population was not applied, and it will be necessary to evaluate the suitability of applying floating population data in the prediction of the spread of infectious diseases in the future. Additionally, there are limitations in predicting the number of infected individuals as population migration among the 25 districts in Seoul was not taken into account. Accordingly, the prediction accuracy of the spread of infectious diseases in Seoul will increase when both floating population data and data of the number of people getting in and out of subways and bus stations are additionally applied to a mathematical model in order to consider interactions among the districts in Seoul.

Nevertheless, this study is meaningful as it visualized changes in the number of infected people on the map, which includes actual geographical information and the changes over time in the number of individuals that belong to S, I, and R classes of population; a mathematical model based on Korea's unique traffic and population movement information could be used. A simulation can be performed on the spread of an infectious disease by applying additional population and traffic data in the future, and the results can be used in making decisions when establishing an effective infectious disease control strategy for each parameter after creating various scenarios before the outbreak of an infectious disease.

\section{Acknowledgments}

This research was supported by the Korea Institute of Science and Technology Information (K-16-L03-C02-S03) 


\section{References}

[1] J. Sachs and P. Malaney, "The economic and social burden of malaria”, Nature, vol. 415, no. 6872, (2002), pp. 680-685.

[2] J. K. Taubenberger and D. M. Morens, "1918 Influenza: the mother of all pandemics", Emerging Infectious Disease, vol. 12, no. 1, (2006), pp. 69-79.

[3] World Health Organization, WHO Global Health Observatory data. (http://www.who.int/gho/hiv/en/), (2016).

[4] World Health Organization, Situation Report ZIKA Virus Disease Yellow Fever Virus Disease. (2006).

[5] R. W. Driggers, C. Y. Ho, E. M. Korhonen, S. Kuivanen, A. J. Jääskeläinen, T. Smura, J. Timofeev, F.J. Rodriguez, L. Levanov, J. Razak, P. Iyengar, A. Hennenfent, R. Kennedy, R. Lanciotti, A. du Plessis and O. Vapalahti, "Zika virus infection with prolonged maternal viremia and fetal brain abnormalities", New England Journal of Medicine, (2016), doi: 10.1056/NEJMoa1601824.

[6] M. G. Roberts and J. A. P. Heesterbeek, "Mathematical Models in Epidemiology", Mathematical Models, Eolss Publishers, Oxford, UK, vol. 3, (2003).

[7] W. O. Kermack and A. G. McKendrick, "A Contribution to the Mathematical Theory of Epidemics", Proceedings of the Royal Society A: Mathematical, Physical and Engineering Sciences, vol. 115, no. 772, (1927), pp. 700-721.

[8] H. W. Hethcote. "The mathematics of infectious diseases", Society for Industrial and Applied Mathematics Review, vol. 42, no. 4, (2000), pp. 599-653.

[9] M. J. Dorfman and J. Medanic, "Scheduling trains on a railway network using a discrete event model of railway traffic", Transportation Research Part B: Methodological, vol. 38, no. 1, (2004), pp. 81-98.

[10] E. Bonabeau, "Agent-based modeling: Methods and techniques for simulating human systems", Proceedings of the National Academy of Sciences., vol. 99, no. 3, (2002), pp. 7280-7287.

[11] W. A. N. G. Jifeng, L.U. Huapu and P. E. N. G. Hu, "System dynamics model of urban transportation system and its application", Journal of Transportation Systems engineering and information technology., vol. 8, no. 3, (2008), pp. 83-89.

[12] E. Pruyt and C. Hamarat, "The influenza A (H1N1) v pandemic: an exploratory system dynamics approach", Proceedings of the 28th International Conference of the System Dynamics Society, Seoul, Korea, (2010).

[13] C. T. Bauch, J. O. Lloyd-Smith, M. P. Coffee and A. P. Galvani, "Dynamically modeling SARS and other newly emerging respiratory illnesses: past, present, and future", Epidemiology., vol. 16, no. 6, (2005), pp. 791-801.

[14] G. M. Hwang, P. J. Mahoney, J. H. James, G. C. Lin, A. D. Berro, M. A. Keybl, D. M. Goedecke, J. J. Mathieu and T. Wilson, "A model-based tool to predict the propagation of infectious disease via airports" Travel Medicine and Infectious Disease, vol. 10, no. 1. (2012), pp. 32-42.

[15] J. Jang and I. Ahn, "Simulation of Infectious Disease Spreading based on Agent Based Model in Korea", Advanced Science and Technology Letters (Healthcare and Nursing 2016), vol.128, (2016), pp.53-58.

\section{Authors}

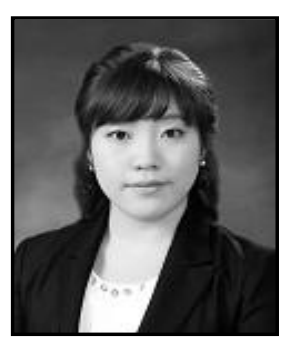

Jinhwa Jang, she is a researcher at Korea Institute of Science and Technology Information in Biomedical Prediction Technology Lab.

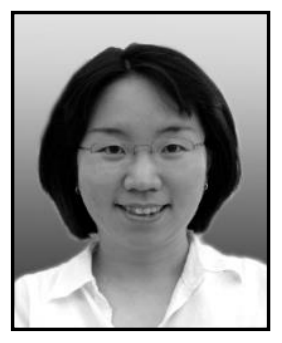

Insung Ahn, she is a principal researcher at Korea Institute of Science and Technology Information in Biomedical Prediction Technology Lab. She is also a professor at University of Science and Technology, KISTI Campus, Department of Big Data Science. 